\title{
Social Well-Being of Iranian Older Adults and its Relationship with Socio-Demographic Variables
}

\author{
Pouya Farokhnezhad Afshar ${ }^{1}$; Mahshid Foroughan ${ }^{2, *}$; AbouAli Vedadhir ${ }^{3}$; Mahmoud Ghazi \\ Tabatabaei $^{4}$ \\ ${ }^{1}$ PhD Candidate in Gerontology, Iranian Research Center On Aging, University of Social Welfare and Rehabilitation Science, Tehran, Iran \\ ${ }^{2}$ Iranian Research Center of Aging, University of Social Welfare and Rehabilitation Science, Tehran, Iran \\ ${ }^{3}$ Faculty of Social Science, Department of Anthropology, University of Tehran, Tehran, Iran \\ ${ }^{4}$ Faculty of Social Science, Department of Demography \& Population Studies, University of Tehran, Tehran, Iran \\ * Corresponding author: Mahshid Foroughan, Iranian Research Center of Aging, University of Social Welfare and Rehabilitation Science, Tehran, Iran. Email: m_foroughan@yahoo.com
}

Received: 22 Sep 2016

Accepted: 21 Nov 2016

Ppub: 23 Dec 2016

\begin{abstract}
Background: Health consists of physical, psycho and social well-being. Social well-being in individual level is a combination of psychological well-being about community. The importance of social well-being is high among the older adults for a different reason such as social isolation and loneliness.

Methods: This is a cross-sectional study which was conducted in Tehran, capital of Iran, in 2016. The participants were communitydwelling older adults selected by using the multistage cluster sampling strategy across the city. We used Social Well-being Scale (SWS) for collecting data.

Results: Three hundred older adults completed the scale. Mean age was $66.39 \pm 6.98$ years; $60 \%$ were male. The data revealed that the older participants were at moderate level in terms of social well-being. Social well-being in these older people was significantly related to their marital status $(\mathrm{P}=0.03)$, level of education $(\mathrm{P}=0.03)$, employment status $(\mathrm{P}<0.01)$, housing status $(\mathrm{P}<0.01)$, financial $(\mathrm{P}<0.01)$ and health status $(\mathrm{P}<0.01)$.

Conclusions: Social well-being in older people is linked to having pension, house ownership, being employed, prospers financial status, education, good health and married status.

Keywords: Social Well-Being; Older Adults; Health
\end{abstract}

